\title{
Depinning of interacting particles in random media
}

\author{
Stefano Zapperi \\ PMMH-ESPCI, 10 rue Vauquelin, 75231 Paris Cedex 05, France \\ and INFM sezione di Roma 1, Dipartimento di Fisica, Università “La Sapienza,'” P.le A. Moro 2, 00185 Roma, Italy \\ José S. Andrade, Jr. \\ PMMH-ESPCI, 10 rue Vauquelin, 75231 Paris Cedex 05, France \\ and Departamento de Física, Universidade Federal do Ceará, 60451-970 Fortaleza, Ceará, Brazil \\ Josué Mendes Filho \\ Departamento de Física, Universidade Federal do Ceará, 60451-970 Fortaleza, Ceará, Brazil
}

(Received 24 November 1999)

\begin{abstract}
We study the overdamped motion of interacting particles in a random medium using the model introduced by Pla and Nori [Phys. Rev. Lett. 67, 919 (1991)]. We investigate the associated depinning transition by numerical integration of the equation of motion and show evidence that the model is in the same universality class of a driven elastic chain on a rough substrate. We discuss the implications of these results for flux line motion in type-II superconductors.
\end{abstract}

\section{INTRODUCTION}

The dynamics of flux lines in type-II superconductors has attracted a wide interest in the past years. ${ }^{1}$ Besides the study of the equilibrium properties of the vortex lattice, much attention has been devoted to the magnetization behavior out of equilibrium, i.e., when the magnetic flux enters the superconductor. Several interesting dynamical phenomena have been observed experimentally, such as hysteresis, ${ }^{2}$ avalanches, ${ }^{3}$ flux front roughening, ${ }^{4}$ and fractal growth. ${ }^{5}$ These features are reminiscent of dynamic critical phenomena commonly observed in disordered systems, as, for instance, in interfaces at the depinning transition. 6,7

Molecular dynamics has been largely used as a modeling technique to simulate nonequilibrium flux-line propagation in terms of interacting particles in a random pinning field. ${ }^{8-12}$ The particles interact through a long-range repulsive force that can be computed from electrodynamics and can be trapped by pinning centers. A vast literature has been devoted to these models in $d=1$ (Refs. 9 and 10) and $d=2,{ }^{8,11,12}$ with different boundary conditions and external driving modes. Several features seen in experiments have also been reproduced.

In this paper, we study the model introduced by Pla and Nori ${ }^{9}$ and analyze its critical behavior. In particular, we study the time dependence of the average velocity of the particles as a function of a constant applied force. We observe a typical depinning transition and analyze the associated critical behavior using finite size scaling methods. Our results indicate that the model is in the same universality class of elastic strings in random media. $6,7,13,14$

\section{MODEL}

In a type-II superconductor, when the external magnetic field reaches $H_{c 1}$, ${ }^{15}$ the magnetic flux penetrates in the material in the form of flux lines. Before equilibrium is reached, the front of flux lines propagates only partially, giving rise to a flux profile denoted as the Bean state. ${ }^{16}$ Under the effect of an external current or because of an imposed flux gradient, the lines will move displaying interesting dynamical behavior. In the simplest case of an infinitely long cylinder, flux lines can be treated as a set of interacting particles moving in a random pinning landscape and can thus be conveniently studied by molecular-dynamics simulations. ${ }^{8-11}$

Two main contributions are important to describe the overdamped dynamics of flux lines in a type-II superconductor: the repulsive interaction among flux lines and their short-range attractive interaction with pinning centers. The equation of motion for each flux line $i$ can then be written as

$$
\Gamma \vec{v}_{i}=\sum_{j} \vec{J}\left(\vec{r}_{i}-\vec{r}_{j}\right)+\sum_{p} \vec{G}\left[\left(\vec{R}_{p}-\vec{r}_{i}\right) / l\right]
$$

where $\Gamma$ is the viscosity. The first term on the right corresponds to the vortex-vortex interaction. The sum is over all other vortices $j$ and $\vec{J}(\vec{r})=\Phi_{0}^{2} /\left(8 \pi \lambda^{3}\right) K_{1}(|\vec{r}| / \lambda) \hat{r}$, where the function $K_{1}$ is a Bessel function decaying exponentially for $|\vec{r}|>\lambda, \lambda$ is the London penetration length, and $\Phi_{0}$ is the magnetic quantum flux. ${ }^{15}$ The second term on the right-hand side accounts for the interaction between pinning centers (modeled in Ref. 9 as localized traps) and flux lines. Here, $\vec{G}$ is the force due to a pinning center located at $\vec{R}_{p}, l$ is the range of the wells (typically $l \ll \lambda)$, and $p=1, \ldots, N_{p} \quad\left(N_{p}\right.$ is the total number of pinning centers). Models of this kind have been used to simulate in great detail the propagation of flux lines in one and two dimensions, and for different forms of disorder, boundary conditions, and external driving modes. $^{9-11}$ In the present study, we will concentrate on a simple realization of this model in $d=1 .{ }^{9}$ Assuming that the force between particles and the interaction between particles and pinning centers can be approximated by piecewise linear functions, the equation of motion for particle $i$ becomes 


$$
\begin{aligned}
\Gamma \frac{d x_{i}}{d t}= & F-A \sum_{j}\left(x_{j}-x_{i}\right) \theta\left(\left|x_{i}-x_{j}\right|-l_{f}\right) \\
& +B \sum_{p}\left(x_{p}-x_{i}\right) \theta\left(\left|x_{i}-x_{p}\right|-l\right),
\end{aligned}
$$

where $l_{f}$ is the range of particle interactions, which should be of the order of $\lambda, A$ and $B$ are the strengths of interaction, and $F$ is an externally applied force.

When periodic boundary conditions are imposed, the model displays a depinning transition as a function of $F$. ${ }^{9}$ For $F<F_{c}$, the particles are trapped, while for $F>F_{c}$, the average particle velocity is nonzero. Close to the depinning transition, the particle velocity responds with avalanches to small changes in the applied force. ${ }^{9}$ Finally, when simulations are performed with $F=0$ and a constant density of flux lines at the boundaries, the model shows a nonlinear Bean profile, ${ }^{10}$ which has been compared with experiments. ${ }^{2} \mathrm{We}$ note that these features are common to two-dimensional simulations. ${ }^{11}$ In this case, however, a much richer phenomenology, related to the formation of plastic channels for flow, can be observed. ${ }^{12}$

\section{SINGLE-PARTICLE PROBLEM}

As an illustration of the behavior of the model, we first analyze the single-particle problem. Even with this simple system, we show that one can already obtain some qualitative information on the effect of the microscopic pinning field on the particle dynamics.

The equation of motion for a single particle is given by

$$
\Gamma \frac{d x}{d t}=F+B \sum_{p}\left(x_{p}-x\right) \theta\left(\left|x-x_{p}\right|-l\right) .
$$

When the particle is not interacting with a pinning center, it moves with constant velocity $F / \Gamma$. The following equation describes the motion of the particle as it enters in the range of a pinning center well [i.e., $\left.x(t=0)=x_{p}-l\right]$ :

$$
\Gamma \frac{d x}{d t}=F+B\left(x_{p}-x\right) \quad x<x_{p}+l
$$

with the solution given by

$$
x(t)=x_{p}+F / B-(F / B+l) e^{-t / \Gamma} .
$$

For $F<l B$ and in the limit $t \rightarrow \infty$, the particle remains trapped, while for $F>l B$, the particle escapes the pinning center, so that we can set $F_{c}=l B$. The force-velocity diagram can then be computed by noting that, for $F>l B$, the particle spends in each trap a time $\tau$ given by ${ }^{17}$

$$
\tau=\Gamma \ln \left[\left(F+F_{c}\right) /\left(F-F_{c}\right)\right] .
$$

The total time $T$ to cross a system of length $L$ with $N_{p}$ pinning centers is given by

$$
T=N_{p} \tau+\left(L-2 l N_{p}\right) \Gamma / F,
$$

so that the average velocity of the particle can be calculated as

$$
v_{s} \equiv \frac{L}{T}=\frac{F}{\Gamma\left\{F n_{p} \ln \left[\left(F+F_{c}\right) /\left(F-F_{c}\right)\right]+\left(1-2 \ln _{p}\right)\right\}},
$$

where $n_{p}=N_{p} / L$ is the number of pinning centers per unit length. We expect this result to be qualitatively correct at low particle densities and far enough from the depinning transition. Close to the depinning transition $F_{c}$, the velocity increases $\operatorname{logarithmically} v_{s} \sim-1 / \ln \left(F_{c}-F\right)$. In the next section, we will show that, when the repulsion between particles is taken into account, the behavior is changed to $v_{s} \sim\left(F_{c}\right.$ $-F)^{\beta}$, in analogy with the depinning transition in other systems.

\section{COLLECTIVE EFFECTS: NUMERICAL RESULTS}

Although the dependence of the depinning force on various model parameters in the one-dimensional model has been extensively studied in the original work by Pla and Nori, ${ }^{9}$ the scaling relation between velocity and force, a characteristic of the depinning transition, has not been investigated there.

Here, we perform the numerical integration of Eq. (2) in order to study the associated depinning transition. We test the effect of system size simulating systems with $N$ $=50,100,200,400$ particles and $N_{p}=10,20,40,80$ pinning centers, respectively. We use periodic boundary conditions and a system of length $L=N / 100$ in order to keep constant both the particle and the pinning center densities. The width of the pinning potential is $l=10^{-3}$ and its strength is $B$ $=10^{3}$, so that the pinning force for the single particle problem is $F_{c} \equiv l B=1$. In all simulations, the particle-particle interaction is characterized by fixing $l_{f}=2 \times 10^{-2}$ and $A$ $=5$. Finally, the results are averaged over several realizations $n_{r}$ of the disorder $\left(n_{r}=500\right.$ for $N=50, n_{r}=50$ for $N$ $=100,200$, and $n_{r}=20$ for $N=400$ ).

We study the decay of the average particle velocity as a function of time $\langle v(t)\rangle \equiv\left\langle\Sigma_{i} v_{i}\right\rangle$, where the average is taken over $n_{r}$. For large forces, $\langle v(t)\rangle$ reaches a finite steady value $v_{s}>0$, while for small forces, $v_{s}=0$. The accurate evaluation of $F_{c}$ is very important since even a small error in its value can strongly affect the determination of the scaling exponents. As a standard practice in the study of absorbing state phase transitions and other dynamical critical phenomena, ${ }^{18}$ we determine $F_{c}$ using finite-size scaling, analyzing the decay of $\langle v(t)\rangle$ for different values of $F$ and $N$.

In Fig. 1, we show the decay of $\langle v(t)\rangle$ for a system with $N=400$ particles and different values of $F$. We see that there is a drastic change of behavior between $F=0.515$ and $F$ $=0.514$. In addition, we study the behavior of $\langle v(t)\rangle$ for these values of the applied force as a function of $N$. Figure 2 shows that the velocity reaches a steady value $v_{s}$ independent of $N$ for $F=0.515$, while $v_{s}$ decreases as $1 / N$ for $F$ $=0.514$. We thus conclude that $F_{c} \simeq 0.5145$. In Fig. 3, we plot the value of $v_{s}$ as a function of $F-F_{c}$ and observe a power-law behavior

$$
v_{s} \sim\left(F-F_{c}\right)^{\beta} .
$$

The best fit to the data yields $\beta=0.22 \pm 0.05$. We note that this value is consistent with $\beta \simeq 0.25$ found in numerical 


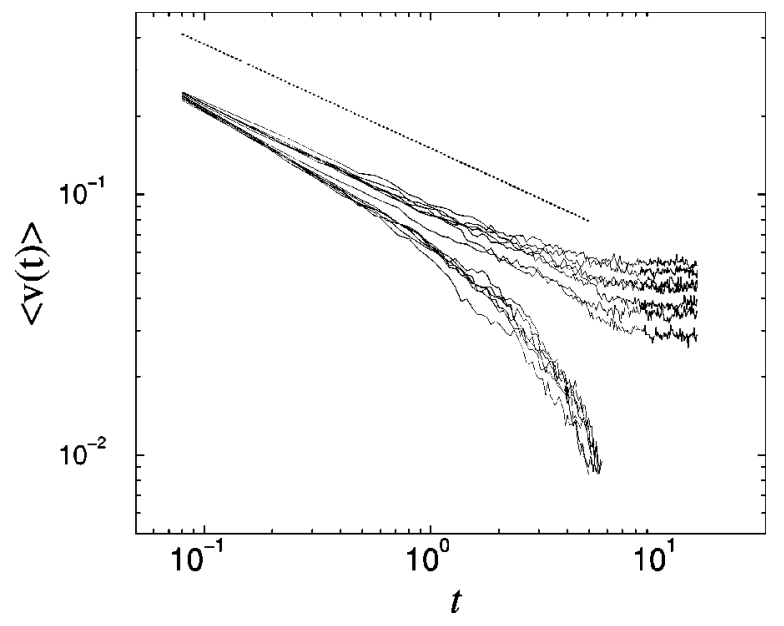

FIG. 1. Log-log plot of the decay of the average velocity with time for a system of $N=400$ particles and $N_{p}=80$ pinning centers. The velocity is averaged over 20 realizations of the disorder. The applied forces range from $F=0.550$ to $F=0.500$ (from top to bottom) and the sharp crossover occurs between $F=0.515$ and $F$ $=0.514$. The dotted line has a slope of -0.4 .

simulations of driven elastic chains on disordered substrate $^{13,14}$ and in the depinning of interfaces in random media. $^{6}$

Finally, we observe that, close to $F_{c}$, it is possible to fit the decay of the average velocity using a power law, $\langle v(t)\rangle \sim t^{-\theta}$, with $\theta \simeq 0.4$. This exponent is conventionally measured in absorbing state phase transitions, ${ }^{18}$ but to our knowledge it has not been estimated at the depinning transition. Using scaling relations, one can readily show that $\theta$ $=z \beta / \nu$, where $\nu$ determines the scaling of the correlation length [i.e., $l_{c o r r} \sim\left(F-F_{c}\right)^{-\nu}$ ] and $z$ is the dynamic exponent relating the correlation length to the correlation time, $t_{\text {corr }}=l_{\text {corr }}^{z}$. The renormalization-group results for driven interfaces $^{6,7}$ gives $\theta=4 / 9 \simeq 0.44$ (using $\nu=1, z=4 / 3$, and $\beta$ $=1 / 3$ ), but the numerical simulations ${ }^{6,13}$ seem to imply a lower value of $\theta \simeq 0.36(\nu \simeq 1, z \simeq 1.42$, and $\beta \simeq 0.25)$. In view of the limited scaling regime observed in our simulations and the absence of direct measures of $\theta$ for interface depinning, we cannot still draw firm conclusions about this point.

\section{DISCUSSION}

In this paper, we have analyzed the depinning transition of interacting particles in a random force field, using the model developed by Pla and Nori ${ }^{9}$ for flux-line dynamics in type-II superconductors. We first noticed that the model displays a depinning transition in the dilute limit, when interactions between particles can be neglected. In this case, the force-velocity diagram is logarithmic close to the transition.

Numerical simulations are then used to investigate the role of interactions on the transitions. In order to obtain an accurate phase diagram, we have used finite-size scaling methods similar to those used to analyze absorbing state phase transitions. ${ }^{18}$ The force-velocity diagram is turned into a power law, as it is commonly found in other depinning transitions. ${ }^{6}$ The critical exponent appears to be in numerical agreement with the one computed for elastic strings in ran-
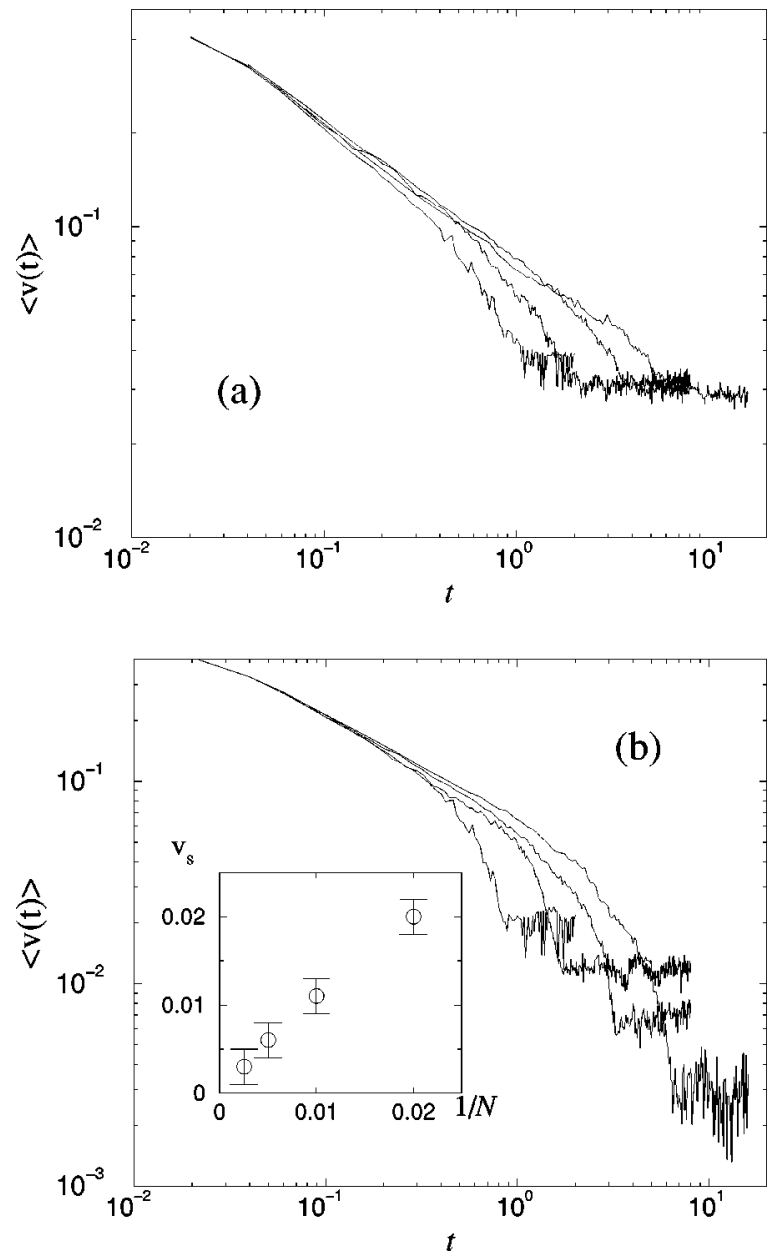

FIG. 2. Log-log plot of the decay of the average velocity with time for systems of $N=50,100,200,400$ particles and $N_{p}$ $=10,20,40,80$ pinning centers, respectively. The velocity is averaged over 50 (20) realizations of the disorder for $N=50,100,200$ (400). The applied force is (a) $F=0.515$ and (b) $F=0.514$. In the inset we show that the steady-state velocity scales as $1 / N$, as it is expected for a subcritical state.

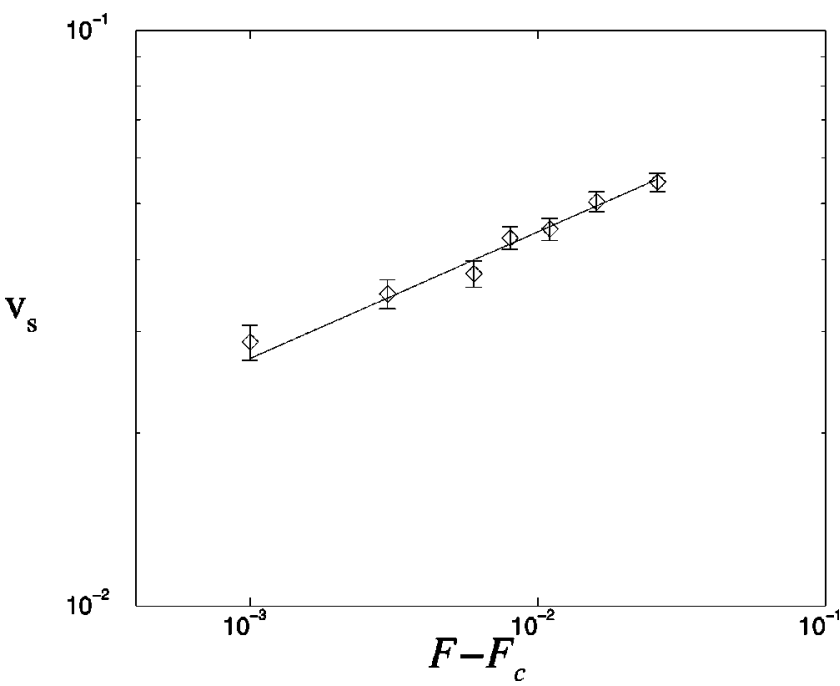

FIG. 3. Log-log plot of the steady-state average velocity as a function of the reduced force $F-F_{c}$, with $F_{c}=0.5145 \pm 0.0005$. The best fit to the data gives $\beta=0.22 \pm 0.05$. 
dom media ${ }^{13,14}$ or "linear" interface depinning. ${ }^{6}$ It would be interesting to compare these two classes of models in two dimensions and check whether universality also holds in this case.

Plastic flow, a phenomenon that is not present in one dimension, could possibly change the behavior of such interacting particle models, since at the depinning threshold the dynamics occurs through well localized channels. This behavior can accurately be reproduced with two-dimensional versions of the model studied here, as the literature shows, ${ }^{11,12}$ but analytical results are difficult to obtain for the plastic case.

In the parameter region corresponding to elastic depinning (i.e., a collective and homogeneous motion), we expect to observe universality with driven interfaces. This phenomenon has been widely studied numerically and by renormalization-group methods, ${ }^{6,7}$ so that exponents are known with good precision. In particular, the exponents for avalanche scaling obtained in this framework could be compared with those measured in the experiments on type-II superconductors. ${ }^{3}$ Similarly, flux front roughening ${ }^{4}$ could be studied in the same way. In order to carry out this investigation, however, it is necessary to understand the precise nature of the boundary conditions and driving modes in the experiments, since, for instance, boundary scaling can be different from the bulk scaling studied here. Finally, the analysis performed here could be directly compared with current-voltage curves measured in type-II superconductors. ${ }^{19}$

\section{ACKNOWLEDGMENTS}

We thank CNPq and FUNCAP for financial support. S.Z. wishes to thank the Physics Department of UFC, where this work has been initiated, for the hospitality.
${ }^{1}$ G. Blatter, M.V. Feigel'man, V.B. Geshkenbein, A.I. Larkin, and V.M. Vinokur, Rev. Mod. Phys. 66, 1125 (1994).

${ }^{2}$ Y.B. Kim, C.F. Hempstead, and A.R. Strnad, Phys. Rev. 129, 528 (1963).

${ }^{3}$ S. Field, J. Witt, F. Nori, and X. Ling, Phys. Rev. Lett. 74, 1206 (1995); C. M. Argenter, Phys. Rev. E 58, 1438 (1998); K. Behnia, C. Capan, D. Mailly, and B. Etienne, ibid. 61, R3815 (2000).

${ }^{4}$ R. Surdeanu, R.J. Wijngaarden, E. Visser, J.M. Huijbregtse, J. Rector, B. Dam, and R. Griessen, Phys. Rev. Lett. 83, 2054 (1999).

${ }^{5}$ R. Surdeanu, R.J. Wijngaarden, B. Dam, J. Rector, R. Griessen, C. Rossel, Z.F. Ren, and J.H. Wang, Phys. Rev. B 58, 12467 (1998).

${ }^{6}$ H. Leschhorn, T. Nattermann, S. Stepanow, and L.H. Tang, Ann. Phys. (Leipzig) 6, 1 (1997).

${ }^{7}$ O. Narayan and D.S. Fisher, Phys. Rev. B 48, 7030 (1993).

${ }^{8}$ H.J. Jensen, A. Brass, and A.J. Berlinsky, Phys. Rev. Lett. 60, 1676 (1988).

${ }^{9}$ O. Pla and F. Nori, Phys. Rev. Lett. 67, 919 (1991).

${ }^{10}$ R. Richardson, O. Pla, and F. Nori, Phys. Rev. Lett. 72, 1268 (1994).

${ }^{11}$ C. Reichhardt, C.J. Olson, and F. Nori, Phys. Rev. B 58, 6534 (1998); 57, 7937 (1998); Phys. Rev. Lett. 78, 2648 (1997); C. Reichhardt and F. Nori, ibid. 82, 414 (1999); C. Reichhardt, C.J. Olson, J. Groth, S. Field, and F. Nori, Phys. Rev. B 54, 16108
(1996); 56, 14196 (1997); C.J. Olson, C. Reichhardt, and F. Nori, ibid. 56, 6175 (1997).

${ }^{12}$ C.J. Olson, C. Reichhardt, and F. Nori, Phys. Rev. Lett. 80, 2197 (1998).

${ }^{13}$ M. Dong, M.C. Marchetti, A.A. Middleton, and V. Vinokur, Phys. Rev. Lett. 70, 662 (1993).

${ }^{14}$ D. Cule and T. Hwa, Phys. Rev. Lett. 77, 278 (1996); Phys. Rev. B 57, 8235 (1998).

${ }^{15}$ P.-G. de Gennes, Superconductivity of Metals and Alloys (Benjamin, New York, 1966).

${ }^{16}$ C.P. Bean, Rev. Mod. Phys. 36, 31 (1964).

${ }^{17}$ We remark that this slowing down bears some similarities with the behavior observed in granular media and other jammed systems. See V.G. Benza, F. Nori, and O. Pla, Phys. Rev. E 48, 4095 (1993).

${ }^{18}$ R. Dickman, in Nonequilibrium Statistical Mechanics in One Dimension, edited by V. Privman (Cambridge University Press, Cambridge, 1996); G. Grinstein and M A. Muñoz, in Fourth Granada Lectures in Computational Physics, edited by P. Garrido and J. Marro, Lecture Notes in Physics Vol. 493 (SpringerVerlag, Berlin, 1997) p. 223; J. Marro and R. Dickman, Nonequilibrium Phase Transitions in Lattice Models (Cambridge University Press, Cambridge, 1999).

${ }^{19}$ S. Bhattacharya and M.J. Higgins, Phys. Rev. Lett. 70, 2617 (1993). 